\title{
Quantum tomography of a nonlinear photonic circuit by classical sum-frequency generation measurements
}

\author{
Francesco Lenzini ${ }^{1, *}$, Alexander N. Poddubny ${ }^{2,3,4}$, James Titchener ${ }^{4}$, Paul Fisher ${ }^{1}$, Andreas Boes ${ }^{5}$, \\ Sachin Kasture ${ }^{1}$, Ben Haylock ${ }^{1}$, Matteo Villa ${ }^{1}$, Arnan Mitchell ${ }^{5}$, Alexander S. Solntsev ${ }^{4}$, \\ Andrey A. Sukhorukov ${ }^{4}$, and Mirko Lobino ${ }^{1,6}$ \\ 1) Centre For Quantum Dynamics, Griffith University, Brisbane QLD 4111, Australia \\ 2) ITMO University, Saint Petersburg 197101, Russia \\ 3) Ioffe Physical-Technical Institute of the Russian Academy of Science, Saint Petersburg 194021, Russia \\ 4) Nonlinear Physics Centre, The Australian National University, Canberra ACT 2601, Australia \\ 5) School of Engineering, RMIT University, Melbourne VIC 30000, Australia \\ 6) Queensland Micro- and Nanotechnology Centre, Griffith University, Brisbane QLD 4111, Australia \\ * francesco.lenzini@griffithuni.edu.au
}

\begin{abstract}
We propose and demonstrate a new method for the characterization of nonlinear multimode integrated devices that reconstruct the biphoton state produced trough spontaneous parametric down-conversion (SPDC) using classical sum-frequency generation measurements. The proposed method is experimentally demonstrated by predicting the state generated from a multi-channel integrated nonlinear waveguide device.
\end{abstract}

Integration of single-photon sources with optical waveguide networks is a key requirement for the development of large-scale quantum photonics technologies $[1,2]$. In particular the engineering of complex optical quantum states on-chip requires the design and fabrication of networks of linear and nonlinear waveguides where photons can interfere and be manipulated $[3,4]$. A reliable and practical technique for the characterization of this class of devices is essential for integrating them as part of more complex circuits or for improving their performances.

Stimulated emission tomography (SET) was proposed in Ref. [5] as an efficient method for predicting the biphoton state produced from a nonlinear device by the use of only classical detectors and laser sources. This technique takes advantage of the analogy between SPDC and its classical stimulated counterpart, i.e. difference-frequency generation (DFG). However SET becomes a challenging -possibly impractical- experimental task for large optical networks as it requires precise information on the linear light dynamics inside the whole structure [6]. Additionally, the analogy with DFG is strictly valid only in the limit of zero propagation losses [7].

Sum-frequency generation (SFG) -the reverse process of SPDC- was identified in Ref. [7] as the ideal approach for characterizing second-order nonlinear circuits in presence of losses. Nevertheless, the method was formulated only for a single and homogeneous waveguide, posing a stringent restriction for the characterization of more complex devices. In this work, we overcome the limitations of the previous proposals by establishing a rigorous equivalence between biphoton wavefunction and the sum-frequency field generated by classical wave-mixing in the reverse direction of SPDC. Our theory analysis is generalized by the use of the Green-function method [8] and holds for arbitrarily complex second-order nonlinear circuits and in presence of arbitrarily large propagation losses. More importantly, the SFG-SPDC analogy can be expressed in any measurement basis, providing a simple experimental tool for the characterization of any "black-box" $\chi^{(2)}$-nonlinear process.
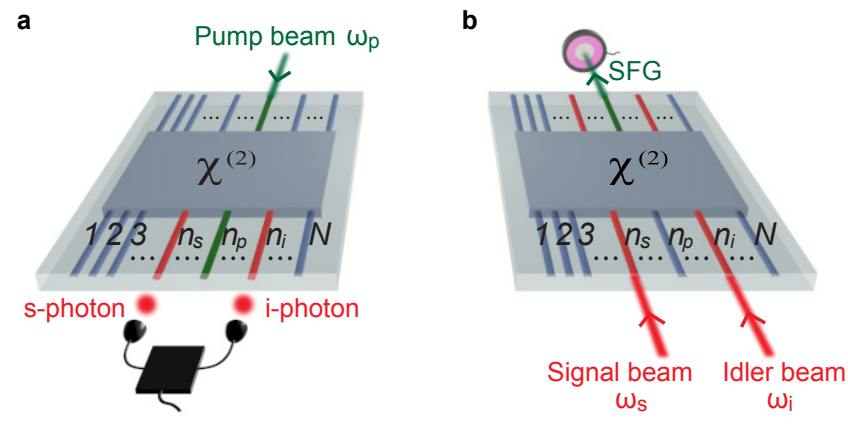

Figure 1: Scheme for the characterization of the biphoton state produced by an array of $N$ waveguides with an arbitrary $\chi^{(2)}$-nonlinear process. a. Schematic of the SPDC process. $\mathbf{b}$. Reconstruction of the biphoton state by SFG measurements.

The proposed characterization method is schematically depicted in Fig. 1 for a waveguide device with $N$ spatial modes with same polarization. A pump beam with frequency $\omega_{p}$ is injected into waveguide $n_{p}$ at the input of the device and produces, by SPDC, the biphoton state (see Fig. 1a)

$|\Psi\rangle_{\text {pair }}=\int_{0}^{\infty} \int_{0}^{\infty} \mathrm{d} \omega_{s} \mathrm{~d} \omega_{i} \sum_{n_{s}, n_{i}} \Psi_{n_{s} n_{i}}^{n_{p}}\left(\omega_{s}, \omega_{i}\right) \mathbf{a}_{n_{s}}^{\dagger}\left(\omega_{s}\right) \mathbf{a}_{n_{i}}^{\dagger}\left(\omega_{i}\right)|0\rangle$,

In the SFG process shown in Fig. 1b, a signal beam with wavelength $\omega_{s}$ and an idler beam with wavelength $\omega_{i}$ (such that $\omega_{s}+\omega_{i}=\omega_{p}$ ) produced from two classical laser sources are injected into waveguides $n_{s}$ and $n_{i}$ in the reverse direction of SPDC. In the limit of no pump depletion, the 
sum-frequency field $E_{n_{s} n_{i}}^{n_{p}}$ generated at the input of waveguide $n_{p}$ is found to be directly proportional to the wavefunction element $\Psi_{n_{s} n_{i}}^{n_{p}}\left(\omega_{s}, \omega_{i}\right)$. This simple analogy can be used to infer the relative squared amplitudes of the wavefunction elements by direct optical power measurements of the generated sum-frequency field. Additionally, the relative phases of the different wavefunction components can be characterized by interferometric measurements of the generated sum-frequency field and used to retrieve the complete biphoton wavefunction.

a
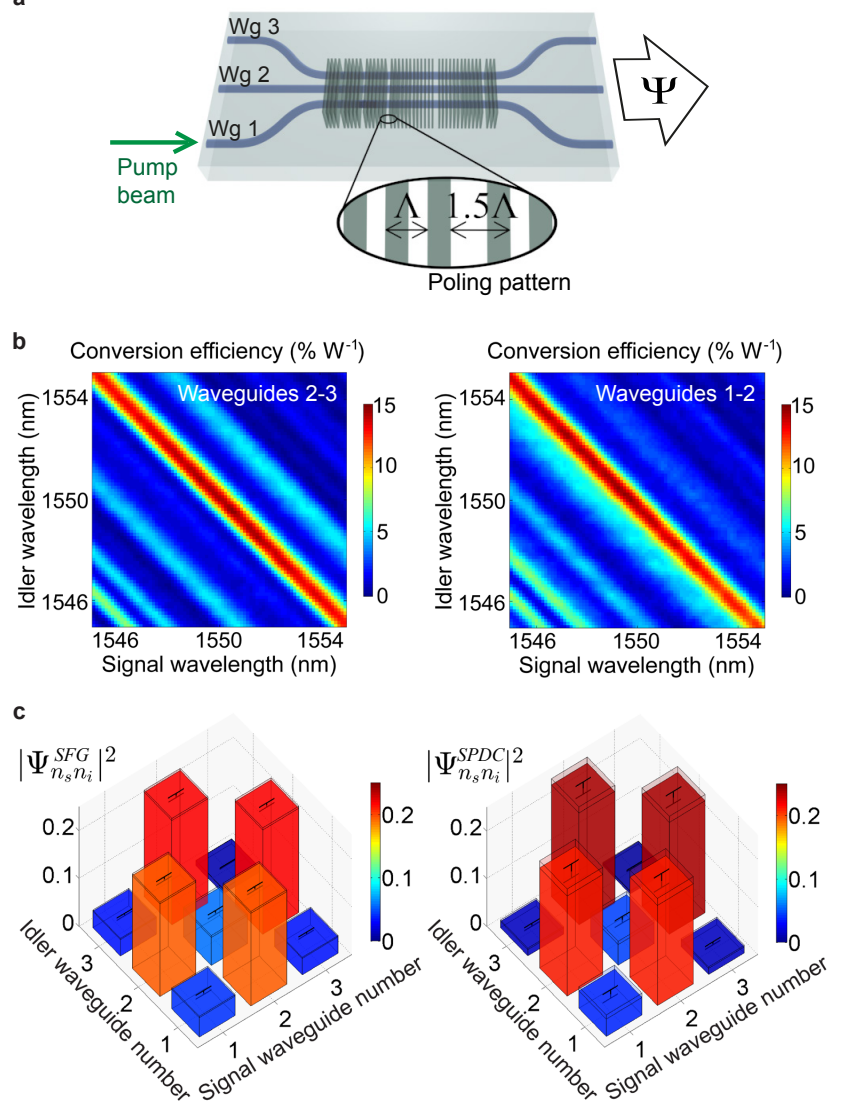

Figure 2: Comparison between SFG and SPDC measurements. a Schematic of the device under characterization and the defect of the periodic poling pattern (inset). b Measured normalized sum-frequency conversion efficiencies at the output of waveguide 1 as a function of signal and idler wavelengths for waveguides 2-3 and waveguides 1-2. d. Comparison between squared relative amplitudes of the wavefunction elements predicted by SFG and measured by SPDC.

The SFG characterization protocol for complex multidimensional systems was demonstrated on an array of nonlinear waveguides based on the recently developed concept of quantum state engineering with specialized poling patterns [6]. The device, schematically depicted in Fig. 2a, is made of three evanescently coupled waveguides fabricated on a z-cut lithium niobate substrate by the use of the reverse proton exchange technique [9]. The three waveguides have an inhomogeneous and asymmetric poling pattern along the propagation direction with 5 defects at different locations of the array introduced by translating the poled domains by half a poling period $\Lambda$. SFG and SPDC measurements are used to test the device when a pump beam is injected into waveguide 1 and directly compared to proof the SPDC-SFG analogy (Fig. 2).

Figure $2 \mathrm{~b}$ shows, as an example, the sum-frequency conversion efficiencies measured at the output of waveguide 1 as a function of signal-idler wavelengths for waveguides 23 and waveguides 1-2 inputs. In both cases we observe a maximum normalized conversion efficiency $\simeq 15 \% \mathrm{~W}^{-1}$ for a sum-frequency wavelength $\left(\lambda_{s}^{-1}+\lambda_{i}^{-1}\right)^{-1}=775 \mathrm{~nm}$. A comparison between the relative squared amplitudes of the wavefunction elements obtained with the two different characterization methods is reported in Fig. 2c. SFG predictions are obtained by measuring the conversion efficiencies for all of the permutations of signal-idler beams in the three waveguides and integrating the data corresponding to a SPDC pump wavelength $\lambda_{p}=775 \mathrm{~nm}$ over a bandwidth of $6 \mathrm{~nm}$. The good agreement between SFG prediction and SPDC measurements results in a fidelity between the two correlation matrices in Fig. 2c $F=\sum_{n_{s} n_{i}} \sqrt{\left|\Psi_{n_{s} n_{i}}^{S F G}\right|^{2}\left|\Psi_{n_{s} n_{i}}^{S P D C}\right|^{2}}=$ $99.28 \pm 0.31 \%$.

In conclusion, we have proposed and demonstrated a practical and accurate method for the characterization of the biphoton state produced trough SPDC in any arbitraryunknown second-order nonlinear process. The method has been experimentally tested by characterizing a complex multi-dimensional system, showing a near-unitary agreement with the results of photon-coincidences counting measurements. Our approach, thus, provides a practical and viable path for the characterization and development of monolithically integrated waveguide networks for large-scale quantum photonics technologies.

\section{References}

[1] O'Brien, J. L., Furusawa, A. \& Vučković, J. Photonic quantum technologies. Nature Photonics 3, 687-695 (2009).

[2] Tanzilli, S. et al. On the genesis and evolution of integrated quantum optics. Laser \& Photonics Reviews 6, 115-143 (2012).

[3] Solntsev, A. S. et al. Generation of nonclassical biphoton states through cascaded quantum walks on a nonlinear chip. Phys. Rev. X 4, 031007 (2014).

[4] Silverstone, J. W. et al. On-chip quantum interference between silicon photon-pair sources. Nat Photon 8, 104-108 (2014).

[5] Liscidini, M. \& Sipe, J. E. Stimulated emission tomography. Phys. Rev. Lett. 111, 193602 (2013).

[6] Titchener, J. G., Solntsev, A. S. \& Sukhorukov, A. A. Generation of photons with all-optically-reconfigurable entanglement in integrated nonlinear waveguides. Phys. Rev. A 92, 033819 (2015).

[7] Helt, L. G. \& Steel, M. J. Effect of scattering loss on connections between classical and quantum processes in second-order nonlinear waveguides. Opt. Lett. 40, 1460-1463 (2015).

[8] Poddubny, A. N., Iorsh, I. V. \& Sukhorukov, A. A. Generation of photon-plasmon quantum states in nonlinear hyperbolic metamaterials. Phys. Rev. Lett. 117, 123901 (2016).

[9] Lenzini, F., Kasture, S., Haylock, B. \& Lobino, M. Anisotropic model for the fabrication of annealed and reverse proton exchanged waveguides in congruent lithium niobate. Opt. Express 23, 1748 1756 (2015). 